\title{
Transformaciones de la estatalidad social en el régimen de acumulación post neoliberal
}

Changes in social statehood in a post neoliberal regime of capital accumulation

Arturo Claudio Laguado Duca Profesor titular del Departamento de Ciencia Política. UNLaM.

Profesor Adjunto del Departamento de Economía, Producción e Innovación Tecnológica. UNPAZ.

alaguado@yahoo.com

\section{Maximiliano Rey}

Profesor Titular Regular del

Departamento de Ciencias Jurídicas y Sociales, UNPAZ.

Profesor Adjunto Regular de la Facultad Ciencias Sociales, UBA

maxirey@hotmail.com
Fecha de recepción:

16.3.16

Fecha de aceptación:

7.8.16

\section{Resumen}

En Argentina, la crisis de 2001 y la redefinición de los postulados neoliberales trajo innovaciones tanto en la sociedad como en el Estado. Uno de los principales cambios fue el nacimiento de un nuevo modo de regulación social, el cual se desarrolló a la par que el Estado tomaba nuevas formas y contenidos. Este artículo se centra en el análisis del nuevo tipo de Estado y de sus intervenciones sociales, observando sus características económicas, políticas y administrativas, pero no en tanto políticas sociales sino como indicadores de la nueva estatalidad y su vinculación con la transformación del modo de regulación.

Palabras clave: Argentina - Estado - estatalidad social - postneoliberalismo - seguridad social - regulación social.

\section{Abstract}

The crisis and subsequent departure from neoliberalism in Argentina brought innovations in the society and the State. One of the major changes was the emergency of a new mode of regulation, which grew up at the same time that the State took new form and substance. This article focuses on the analysis of the new type of State and its social interventions -observing the political, economic and administrative dimensions- but not as social policies but 
as indicators of a new statehood and its relationship with the transformation of the mode of regulation.

Key words: Argentina - state - social statehood - postneoliberalism - social security - social regulation.

\section{Introducción}

El nuevo siglo trajo novedades en la sociedad y la estatalidad argentinas. La relación entre ambas se vio modificada por un nuevo modo de regulación, el cual se desarrolló a la par que el Estado tomaba nuevas formas y contenidos. Uno de estos se verificó en el conjunto de intervenciones sociales del Estado, desde la muy conocida Asignación Universal por Hijo -AUH-, sancionada en 2009, hasta la ampliación de la atención directa del aparato administrativo estatal en los diferentes lugares que componen el territorio nacional.

A la luz de esta situación, en este trabajo adelantamos algunos de los cambios ocurridos en la estatalidad social, no como un objeto de estudio en sí misma sino en estrecha vinculación con la transformación del modo de regulación y sobre todo del tipo de Estado que surgió luego de la crisis de 2001.

Vale decir, en este artículo no haremos una evaluación de la política social de la última década, cuyas virtudes, creemos, ya han sido estudiadas. En su lugar, abordaremos las características de algunas iniciativas del sector de políticas sociales como ejemplo de una concepción diferente de la protección social y por ende de un tipo de Estado distinto al del fin de siglo XX.

Sostenemos que la protección social ejemplifica la relación que una sociedad tiene -en términos ideales- consigo misma. Relación que en última instancia está mediada por el Estado o, mejor, por la legitimidad de su intervención y los límites de su accionar.

Pero la política de protección social no es independiente de una concepción de lo social y del régimen de acumulación en que ella se inserta. Distintas formas de acumulación, históricamente, han sido acompañadas por modos de regulación con los que hacen sinergia. ${ }^{1}$ Una rápida secuenciación histórica nos permitirá demostrar esta afirmación.

Por tanto, no se mirará aisladamente una serie de políticas sino que se la considerará dentro del modo de regulación del que hace parte -brevemente, del tipo de Estado 
en que se enmarca-. Queda pendiente discutir si estas modificaciones de la regulación social incluyen, también, modificaciones en el régimen social de acumulación. No hay duda, sin embargo, y eso es lo que se discute en este trabajo, que la emergencia de los gobiernos postneoliberales en los albores del siglo XXI, implica un nuevo modo de regulación y, con él, el retorno de las formas de estatalidad estadocéntricas.

En todo caso, sostendremos que las políticas sociales emprendidas por los gobiernos postneoliberales en Argentina, forman parte de un tipo de Estado diferente que, a falta de un nombre mejor, podemos llamar Nacional Popular.

\section{La inclusión en perspectiva histórica}

La Seguridad Social es la forma que encontraron los Estados modernos de manejar la contradicción entre la igualdad formal ante la ley y la inevitable desigualdad en el mercado, en las sociedades de clases. Esa contradicción entre igualdad formal y desigualdad de ingresos, o si se quiere, entre sociedad liberal que garantizaba la igualdad ante la ley (derechos políticos) y un orden económico que implicaba el empobrecimiento generalizado, fue el gran desafio que enfrentaron los Estados de Bienestar ${ }^{2}$ del siglo pasado y que fueran teorizados por Marshall (1997) en su texto Ciudadanía y clase social.

El Estado de Bienestar implicó una amplia intervención en la sociedad, o sobre una parte de ella definida como 'objeto de intervención'. Esta intervención, sin embargo, está relacionada con una materia anterior que no siempre se presenta directamente en el debate: los alcances de la solidaridad nacional y las definiciones de ciudadanía que lleva implícita. Desde el siglo XIX, esta preocupación será bautizada como la cuestión social (Donzelot, 1994; Rosanvallon, 1995; Castel, 1997).

Marshall creía que esa contradicción -surgida con la Revolución Francesa-, llevaba siempre latente el riesgo de fractura de la sociedad. Los derechos sociales, es decir aquellos que tenían como objetivo garantizar la autonomía individual al solucionar las necesidades más inmediatas del ciudadano, evitarían ese riesgo al reemplazar el principio clasista por el de la solidaridad ${ }^{3}$ (Donzelot, 1994).

Claro está, la preocupación por la posible ruptura estaba asociada al descubrimiento de las dificultades de legitimación inherentes en las sociedades capitalistas por causa de la mencionada contradicción. Por ello, esa preocupación -enfrentada como la cuestión social del siglo XX- implicará también amojonar los límites de la comunidad política, las calidades para su pertenencia plena y el papel del Estado en la activación de la ciudadanía. En ese sentido, la cuestión social es una cuestión política por excelencia, toda vez que en ella está presente la categoría central de ciudadanía y los derechos que en ésta se articulan. 
El objetivo de la política social es, pues, difuminar esa fractura. Con tal fin, la idea un poco abstracta de política social, se concreta en políticas sectoriales. De esta forma, si la política social -en singular- enfatiza el componente político de las acciones de la sociedad sobre sí misma (Grassi, 2003; Laguado Duca, 2011), las políticas sectoriales son la manera en que se operacionalizan técnicamente.

En las sociedades capitalistas -o sociedades basadas en la salarización, según la expresión de Castel (1997)-, de todas las políticas sociales, las relacionadas con el trabajo ocupan un lugar fundamental. Por ello, desde los primeros pasos de la seguridad social con Bismarck, o en las sociedades que construyeron Estados de Bienestar más o menos maduros, las protecciones se han asociado principalmente al mundo del trabajo... o a su carencia. Ese ha sido el caso de América Latina, y particularmente, el de Argentina. ${ }^{4}$

Siguiendo a Andrenacci y Soldano (2005) -en cuya periodización nos basamos- podemos agrupar la política social en tres grandes ejes

- Políticas de trabajo.

- Políticas de servicios universales: referidas fundamentalmente a la administración de sectores sociales como vivienda, educación, etc.

- Política asistencial: entendida como aquellas intervenciones destinadas a socorrer a la población más vulnerable que no tiene acceso -o lo tienen deficitariamente- al mercado de trabajo.

Sin embargo, como ya se mencionó, la orientación de las políticas sociales depende, en última instancia, del componente político de la política social. Es decir, de la concepción de solidaridad que reina en una sociedad y del papel que se le atribuya al Estado en tanto motor activo en su construcción, en tensión con las iniciativas de valorización del capital. O, para ser más rigurosos, de la manera en que se articulen el modo de regulación y el régimen de acumulación en distintas etapas del capitalismo (Jessop, 1999).

En esa lógica, diferentes etapas del capitalismo implicaron distintas formas de regulación en cada una de estas áreas de política social.

Según Andrenacci y Soldano (2005), destacan durante el período liberal de acumulación -que en Argentina coincidió con la etapa formativa del Estado nacional y se extendió hasta la crisis del modelo agroexportador- las políticas relacionadas con el empleo, centradas en la construcción del mercado de trabajo por la combinación de la represión -ley de Residencia de 1902 y ley de Defensa Social, 1910- y las primeras políticas de protección que sólo cubrían a empleados públicos, como la ley de jubilaciones de 1887 , o la de accidentes de trabajo de 1915. 
Ese período tuvo una política de administración de servicios universales, dirigida en lo fundamental a los sectores medios y trabajadores urbanos. Estas políticas hacían sinergia con el régimen de acumulación, pero también con el objetivo de nacionalizar a la población migrante e incorporar a la población urbana. La modernización urbana se complementó con los primeros pasos en salud pública de la mano del higienismo y con la ley de educación básica de 1880, que se sumaba a este doble objetivo de nacionalizar población y crear una mano de obra moderna.

Las políticas de asistencia, como fue típico de los Estados liberales, se centraron en la gestión de los desafiliados. ${ }^{5}$ La Sociedad de Beneficencia fundada por Rivadavia y la creación hacia 1930 de la Dirección Nacional de Higiene, tuvieron un rol central en este eje.

Esta configuración caracterizada por la tímida intervención estatal se mantendrá hasta que la crisis mundial de 1929 ponga en duda las bondades del modelo de acumulación del país y del modo de regulación que lo acompañó. De la mano de las dificultades del sector agroexportador, se irá legitimando la intervención del Estado hasta lograr una importante presencia durante la década de los cuarenta.

El capitalismo europeo enfrentó la crisis mundial con la extensión del Estado de bienestar. Eso implicó nueva sinergia entre el régimen de acumulación y modo de regulación en lo que algunos autores llamaron Estado de Bienestar Keynesiano -EBK- (Jessop, 1999). ${ }^{6}$

El EBK ha sido suficientemente estudiado para que haga falta destacar aquí sus características. En América Latina, ese Estado de bienestar tuvo sus formas atemperadas en los Estados nacional populares y en los desarrollistas. Dos formas estatales diferentes, con distinta concepción del lugar que debía ocupar la redistribución en el proceso de modernización e industrialización, pero que en esencia respondían a una nueva forma de concebir el papel del Estado -ahora preponderante- en la regulación social (Cao y Laguado Duca, 2014). ${ }^{7}$ Llamaremos estadocéntrica a esta manera de pensar el rol estatal.

En Argentina, las políticas de trabajo ocuparon una centralidad que hacía sinergia con un modelo de Estado que ambicionaba el pleno empleo como sostén del mercado interno, para garantizar el despegue de la industria nacional. La búsqueda del pleno empleo fortaleció aún más la fuerte asociación entre trabajo formal y protección social. Se produjo un consenso sobre la obligatoriedad de la previsión social -recogido tanto por el artículo 37, inc. 7 de la reforma constitucional de 1949, como por el artículo 14bis de la reforma constitucional de 1957- y el movimiento sindical pasó a ser un actor importante para la protección social. Se extendió la regulación pública, ya iniciada durante los gobiernos nacional populares, y se tomaron una serie de medidas para racionalizarla: intento de unificación de Cajas de Previsión (1958); Salario mínimo vital y móvil (1963); Reglamentación de Cajas (1969); Ley 18.610 sobre Obras Sociales (1970), etc. 
configurando una forma de solidaridad fragmentada que caracterizó al desarrollismo argentino (Andrenaci y Soldano, 2005; Laguado Duca, 2011).

Las políticas universales complementaron la asistencia asociada al trabajo formal en una sociedad que tendía hacia la salarización de su población. De esa forma se masificó la educación gratuita en los niveles medio y universitario, se construyó una red pública de hospitales, se expandió la infraestructura, se iniciaron políticas de vivienda social y se estatizaron y universalizaron los servicios públicos en las zonas urbanas y periurbanas.

En ese marco, las políticas de asistencia pasaron a ocupar un lugar residual, sin desaparecer. Orientada, en lo fundamental, a transferir bienes y servicios para aquellos que no lograban integrarse plenamente al mercado de trabajo, la Fundación Eva Perón ocupó un lugar paradigmático a mitad de siglo, posteriormente reemplazada por diferentes formas de apoyo a los desafiliados con fuerte presencia de organizaciones religiosas.

En todo caso, es sabido que a partir de la crisis de la deuda de los años 80 y la posterior implementación de las recetas del Consenso de Washington (Williamson, 1990) en la región latinoamericana, los postulados estadocéntricos -fueran desarrollistas o nacional populares- fueron abandonados. Fue la hegemonía de lo que se conoció como neoliberalismo. En la Argentina correspondió a la reforma del Estado y el ajuste estructural de las décadas del ochenta y noventa. ${ }^{8}$

El Estado neoliberal no sólo fue una importante mutación en el régimen de acumulación ${ }^{9}$-ahora signado por la movilidad de capital, la desregulación y la valorización financiera-, sino también en el modo de regulación. El Estado mínimo no implicó únicamente una relativa retirada de la participación activa en economía, (incluida la desregulación del mercado de trabajo tendiente a la reducción de los costos laborales y, junto con ello, a una remercantilización parcial de las coberturas de aseguramiento) sino también una transformación de los mecanismos de intervención.

La desregulación del mercado de trabajo se plasmó en una amplia legislación: p.e. la Ley Nacional de Empleo, en 1991, permitió la contratación temporal de trabajadores; ese mismo año, la ley 24.028 modificó el régimen de indemnizaciones por despido, la ley de PYMES flexibilizó el trabajo en negro, etc. Todas estas leyes neutralizaron la función protectora del salario mínimo -de fuerte tradición en Argentina-, permitiendo la precarización del empleo y fomentando el cuentapropismo.

Coherentemente, las políticas referidas a los servicios universales pasaron a ser dependientes del lugar que ahora se le otorgó a la lógica macro económica liberal. En momentos de ajuste, la inversión se ató a la reducción del gasto fiscal, lo cual se intentó mediante 
la descentralización de los servicios, poniendo en riesgo en la práctica la obligación del Estado de hacerse cargo de este gasto.

Paralelamente, comenzó un proceso de privatización de los servicios públicos con débil regulación mientras tendieron a crearse cuasimercados (Crojethovic y Ariovich, 2008), por ejemplo, con la ley de Desregulación de las Obras Sociales y la de Hospitales de Autogestión en 1993 o la de Reforma Previsional en 1994.

La política asistencial nuevamente alcanzó un rol protagónico, pero a diferencia de los inicios del siglo XX, se desarrolló bajo el principio de corresponsabilidad público/privado. En un marco de crecimiento y complejización de la pobreza -pobreza estructural, nuevos pobres, población en riesgo- se plantearon simultáneamente dos novedades en tanto estrategia de intervención: la intervención de emergencia para los damnificados del ajuste estructural mediante la focalización de beneficiarios y la tercerización mediante ONGs o entidades sociales para que la ayuda llegue a aquellos que más la necesitan.

Si la focalización -o el subsidio a la demanda, como se la llamó también en lenguaje más duramente económico- fue la novedad más destacada del Estado mínimo en cuanto a las transformaciones en las políticas sociales; la asociación de política social con "programa" -y la aparición del 'beneficiario' como objeto/sujeto de estos programas- fue otra innovación de importancia no menor. Los programas se multiplicaron: Plan trabajar en 1996, Programa de emergencia laboral en 2000, Plan jefas y jefes en 2002, entre muchos otros.

En definitiva, según el pensamiento neoliberal, todo aquello que podía ser gerenciado por el mercado debía quedar por fuera de la intervención estatal. En cambio el Estado debía centrarse en las funciones para lo cual estaba más capacitado: garantizar el respeto a la propiedad, proveer la seguridad física y jurídica, promover las inversiones en infraestructura, y establecer instituciones confiables que favorecieran la atracción de capitales. El resultado más notorio de estas ideas trasladadas a la práctica fue el abandono de los principios solidaristas de la Seguridad Social que habían orientado la tradición argentina.

Al desligar la política social del pleno empleo, el Estado neoliberal rompió la sinergia entre bienestar social y crecimiento económico. El resultado fue que una gran cantidad de población quedó carente de empleo de calidad que asegurase la protección social o, simplemente, carente de empleo. En esa situación el Estado debió hacerse cargo de estos individuos dependientes que ya no encontraron protección asociada al trabajo o, que, por sus condiciones laborales, no tenían capacidad de financiar su retiro futuro. Esta tara la asumió a través de los estados subnacionales (servicios universales) o apelando en gran medida a la tercerización en ONGs (servicios asistenciales) y a una miríada de programas focalizados. 
Si bien es cierto que esta manera de enfocar la previsión social estaba influenciada por la necesidad perentoria de ahorrar recursos en un contexto de crisis de la deuda, en última instancia estas transformaciones se asocian a un nuevo tipo de capitalismo que concibe un lugar muy distinto para la intervención estatal. Se rompe la sinergia producida entre la acumulación fordista y una regulación social que se basaba en sociedades de pleno empleo, del EBK. Pierde sentido, entonces, la asociación de la protección social con el mercado formal de trabajo, o la de ciudadanía social con los derechos laborales e, incluso, la cobertura universal de los servicios que garantizaba el Estado -con mayor o menor eficiencia- con ciudadanía laboral.

La centralidad que vuelve a ganar la asistencia en la política social obliga a conceptualizar este fenómeno desde un ángulo que trascienda el de la eficiencia del gasto público o la muy interesante discusión de la apropiación corporativa del gasto social que, efectivamente, se produjo durante el período desarrollista en Argentina.

En esa misma lógica, para entender la contrarreforma de la intervención social estatal emprendida en el siglo XXI luego del rotundo fracaso del Estado neoliberal, es necesario inscribirla en otro modelo de estatalidad.

\section{El retorno del Estado ${ }^{10}$}

El cuasi derrumbe estatal del 2001 enterró el régimen de Estado mínimo que se había impuesto en Argentina, como en casi toda América Latina. A partir de entonces nacerá un nuevo modelo de Estado cuya definición está aún en discusión entre los especialistas. En todo caso hay consenso en que se abrió una etapa caracterizada por el énfasis en la recuperación simbólica y material del Estado como garante de los procesos de inclusión social y política, renovada capacidad de regulación de los mercados, políticas industrialistas, junto con una marcada vocación latinoamericanista y manifiesta independencia de los centros de poder mundial.

Estudios recientes (Cao y Laguado, 2014) han mostrado la confluencia de dos tradiciones en la emergencia del Estado post neoliberal: el desarrollismo -o su versión contemporánea, el neodesarrollismo- y la tradición nacional popular. ${ }^{11}$ En este apartado haremos una muy esquemática referencia a estas ideas para comprender en ese marco las redefiniciones de la política social.

Retomando elementos del desarrollismo de mitad del siglo XX, el neodesarrollismo vuelve a poner en un lugar central la intervención estatal en la regulación social y la inversión económica, dando un lugar destacado al sector público en el impulso a la $\mathrm{I}+\mathrm{D}+\mathrm{I} .{ }^{12}$ Sin embargo-y como es lógico ante el cambio de los tiempos- también se proponen modificaciones respecto al modelo que primó en el siglo pasado. Por 
ejemplo, se flexibiliza la protección de los derechos del trabajo en un mundo donde la movilidad del capital y la financierización de la economía, debilitan el poder de los Estados nacionales.

Manteniendo el énfasis en el desarrollo industrial y en el fortalecimiento de la demanda agregada como base del take off industrializador, se toma en serio la tradicional restricción externa que azotó la economía argentina por la carencia estructural de dólares, pero sin abandonar el principio de una fuerte intervención estatal y un manejo prudente de las cuentas fiscales (esta tendencia se debilita a partir de la crisis mundial de 2008).

Thwaites Rey lo resume como «dar al Estado el rol de centralizador y asignador de la renta del recurso nacional básico [refiere a hidrocarburos, minería, soja, etc.]; a la sociedad civil se le cede la tarea del desarrollo endógeno y esto se combina con la interpelación a una burguesía nacional, que además de pequeños y medianos empresarios de base local incluiría a empresas grandes y, en particular, a las transnacionales de base regional (las denominadas multilatinas), que han crecido en las últimas décadas en la región» (Thwaites Rey, 2010: 32).

De hecho, si algo caracterizó a la etapa iniciada en 2003, fue un énfasis en la reindustrialización, con el objetivo de reiniciar el círculo virtuoso propuesto por Keynes para reactivar la demanda: pleno empleo, mercado interno, fortalecimiento fiscal, inversión social.

Una serie de medidas se impulsaron en esa dirección. A modo de ejemplo se pueden mencionar el Plan Estratégico Industrial 2020 del Ministerio de Industria, cuyo objetivo fue fortalecer sectores de la industria nacional dedicados a la exportación, o el Proyecto de Promoción de las Exportaciones de Agroalimentos Argentinos -PROARGEX-, iniciativa del Ministerio de Agricultura, Ganadería y Pesca y del Banco Interamericano de Desarrollo.

En la práctica, hasta que la reciente crisis mundial golpeara a las economías latinoamericanas, la tasa de crecimiento industrial estuvo cerca del 10\% (CENDA, 2010). Estas medidas no lograron, sin embargo, revertir la extranjerización de la economía, ni la débil integración vertical de la industria nacional. De esta forma, la restricción externa se mantuvo presente, obligando a controlar la fuga de divisas al exterior, acelerada por la revaluación del peso que jaqueó el gran instrumento industrializador del gobierno: el cambio competitivo.

Para lo que nos interesa -la caracterización del Estado posneoliberal- independientemente de los dilemas económicos que enfrentó, el Estado retomó su papel de agente activo en la regulación económica y, cuando fue necesario para proteger el mercado interno o la balanza de pagos, en la inversión directa vía reestatización de empresas. 
La otra vertiente que influirá en modelo estatal posneoliberal, es la tradición nacional popular, actualizada en el siglo XX. El punto de vista Nacional Popular tiene una perspectiva situada, tanto en lo histórico como en lo geográfico. Hace centro en nuestro país como parte de una nación en construcción -América latina- cuyo proceso histórico puede leerse en clave de búsqueda de plena autonomía y de superación de los desequilibrios de su estructura social. Se trata de un espacio que la visión nacional y popular asume como en desarrollo, con marcados desequilibrios políticos, económicos y sociales: asimetría entre el poder regulador del sector público y los segmentos más concentrados de la economía, agudas desigualdades territoriales, funcionamiento restringido de su institucionalidad (para las formas y estándares canonizados por el establishment), bolsones de marginalidad social, sobredimensión del poder relativo de fracciones del capital internacionalizadas, debilidad de los encadenamientos productivos, etc. La situación periférica y la dinámica del capitalismo mundial, como así también las tensiones que se generan en su propio territorio, alimentan tendencias hacia la reproducción de estos desequilibrios.

En este marco, el objetivo último de la actividad política -y de la acción del Estado como instrumento privilegiado de dicha actividad- es salir de la situación de subdesarrollo. Superarlo implica abandonar el lugar periférico y dependiente en la economía mundial y sortear los desequilibrios sociales, con especial énfasis en la tarea de operar sobre los bolsones de pobreza y marginalidad, así como de reducir los desniveles en la asignación del ingreso. Estos dos objetivos se consideran estrechamente relacionados: sólo una sociedad que haya superado sus desequilibrios sociales puede desarrollarse; sólo a través del desarrollo pueden superarse los desequilibrios sociales.

Pero, la centralidad que tiene el significante justicia social en esta tradición, explica que las normas distributivas promulgadas con posterioridad al 2003 trasciendan con mucho la generación de bienes sociales -meritory goods- propuesta por el desarrollismo.

En esa lógica, se llevó a cabo una importante agenda redistributiva como Plan SUMAR, Procrear, Remediar, Argentina Trabaja, Microcréditos, Manos a la Obra, Conectarigualdad, entre otras, dirigida a la ampliación de derechos. ${ }^{13}$

Es importante destacar, que estas iniciativas se inscriben en otra concepción de Estado, que reinstala su responsabilidad en la (re)producción del tejido social. Abundante legislación post 2003 recupera el principio de solidaridad colectiva que caracterizó a los Estados de Bienestar de postguerra y al primer peronismo en la Argentina.

Por otro lado, en buena parte del resto del Estado, la realización de inversiones en infraestructura largamente retrasadas, y el incremento del gasto corriente - p.e. mejoramiento de dotaciones y sueldos, gastos en bienes de uso- han permitido que el sector público muestre un rendimiento y cobertura superior al que se observó durante los 90 . 
En definitiva, la visión nacional popular sostiene que los elementos que configuran el desarrollo -creación de más riqueza acompañada de nuevos y más complejos roles sociales, incorporación de tecnología, distribución de riqueza, entre otros- no surgen sin más del automatismo de mercado, sino que precisan de una serie de acciones dirigidas y conscientes que involucran al Estado y la Administración Pública.

\section{La reconfiguración de la estatalidad social ${ }^{14}$}

Además de la nueva presencia reguladora, el Estado verifica un cambio en el modo de su intervención en cuatro grandes ejes: universalización, integralidad, territorialización y protagonismo popular.

Como sostuvimos en la Introducción, no nos abocamos al análisis de las iniciativas de política social en cuanto tales sino que las observamos en tanto expresión de un tipo de Estado.

En tal sentido, haremos foco en características de diferentes acciones que componen la estatalidad social postneoliberal. Por tal entendemos un conjunto de atributos relativos a la intervención social del Estado, que lo conforman institucionalmente pero en estrecha relación con su rol como instancia de dominación política, que le exige tomar parte de diversas formas -la social es una de ellas- del proceso de construcción social. ${ }^{15}$

En este específico sentido, ya sean programas como el Conectar-igualdad, principios que dan lugar a modos de gestión como la integralidad, formas de relacionarse con la sociedad como la territorialización o el protagonismo social o grandes políticas universales como la AUH; todas muestran una forma -de intervención social- propia del Estado descripto en la sección previa.

\section{Tendencia a la universalización}

La AUH es una de las iniciativas más destacadas de la segunda mitad de la década pasada, tanto por su innovación como por su quiebre fuerte con la focalización, en base a su tendencia a la universalización. ${ }^{16}$

La página de la Administración Nacional de Seguridad Social define a la AUH como

un derecho que les corresponde a los hijos de las personas que están desocupadas, trabajan en la economía informal con ingresos iguales o inferiores al Salario Mínimo, Vital y Móvil, monotributistas sociales, trabajadores del servicio doméstico, trabajadores por temporada en el período de reserva del puesto o perciban alguno de los siguien- 
tes planes [...]. El cobro de la Asignación Universal por Hijo requiere la acreditación anual de escolarización y controles de salud de los niños. Se abona a los menores de 18 años, hasta un máximo de 5 hijos, priorizando a los hijos discapacitados y a los de menor edad. Se liquidará a uno solo de los padres priorizando a la mamá. En el caso de un hijo discapacitado, el cobro de la asignación es sin límite de edad, y se liquida un monto especial. Para percibirla, deberá tramitar previamente la autorización para el pago de asignaciones por hijo con discapacidad emitida por ANSES (ANSES, s/f).

La prestación monetaria que otorgó inicialmente la AUH se fijó en monto equivalente a la que corresponde a la mayor asignación por hijo del régimen de asignaciones familiares contributivo nacional. Luego, la ley que aprobó el Congreso Nacional, fijaría retribuciones mayores atadas a una fórmula de indexación automática.

Hacia 2010, la asignación cubría al 36.6\% de los menores que, sumados al 53.3\% que cobija el régimen contributivo, indicaría que el $90 \%$ de ellos percibirían asignaciones familiares. El 7,2\% de los menores pertenecientes a familias de ingresos medios y altos, reciben el beneficio indirectamente, puesto que pueden deducir del impuesto a las ganancias una suma por cada hijo a cargo. La reciente promulgación de la asignación por embarazo, extiende el beneficio al período de gestación.

A un año de la puesta en marcha de la AUH, la Central de Trabajadores Argentinos consideraba que era significativo su impacto en la reducción de la pobreza, existiendo una fuerte concentración del beneficio en los hogares más necesitados (CIFRA, 2010).

Más allá del consenso sobre los logros de la AUH, de su innegable éxito para atacar algunas situaciones de exclusión producidas por la crisis del mercado de trabajo, nos interesa contextualizarla en otro modelo de Estado.

Las asignaciones familiares, atadas al trabajo formal tal como las concebía el Estado desarrollista, sufrieron un colapso con los ajustes de la última década del siglo XX y la crisis de 2001, tras la cual el índice de desocupación rondó entre el 22\% y el 25\% de la PEA según la fuente. Aun cuando la gran creación de empleo en los años siguientes a la crisis modificó en parte este escenario, ${ }^{17}$ la AUH se constituyó en una forma de ampliar la cobertura del sistema de seguridad social -recuperado por el Estado con la reestatización del sistema de pensiones privado- reconociendo las situaciones de exclusión todavía existentes. Sin embargo, la AUH no puede ser considerada solamente un programa de transferencias condicionadas más exitoso que otros. Debe entenderse 
en el marco de la emergencia de un nuevo modelo de regulación: el de los gobiernos Nacionales Populares que surgen en el siglo XXI en América Latina.

Una característica fuerte de ello, es tender a la universalización de la seguridad social -como es el caso de la AUH en tanto mecanismo para extender el derecho a las pensiones familiares para los hijos de los trabajadores no formales- retomando los aspectos solidaristas que caracterizaron a los Estado de Bienestar y la tradición nacional popular de mitad del siglo XX. La recuperación de la tradición solidarista en las políticas de prevención no es un hecho aislado. Éstas hacen parte de la naturaleza del Estado activo, al igual que la tendencia a la universalidad y a la integralidad (Cao y Laguado, 2014). ${ }^{18}$

Es importante destacar la universalidad de las políticas más allá de las contemporáneas discusiones sobre la relación costo beneficio de éstas, pues implican la ampliación de los derechos sociales y con ellos, la desemercantilización de aspectos de la reproducción social, en una tradición que ha sido cara a los Estados de Bienestar pero también a los regímenes nacional populares latinoamericanos. ${ }^{19}$

\section{Tendencia a la integralidad}

El principio de integralidad implica abordar los fenómenos complejos desde sus múltiples aristas. De tal modo, las iniciativas del área social no pueden pensarse como programas estancos sin conexión entre sí, sino como acciones coordinadas destinadas a tratar los diversos determinantes de una problemática dada. Por tanto, las diferentes iniciativas de la política social deben pensarse para que se refuercen mutuamente i.e. integralmente.

Esta característica se pudo observar (en mayor o menor grado y con más o menos éxito según los casos) tanto entre ministerios como al interior de cada cartera.

Apuntando a que este principio sea el eje rector de las Políticas Sociales, desde el inicio de la gestión del gobierno de Néstor Kirchner se reconvirtieron las iniciativas programáticas del Ministerio de Desarrollo Social, en orden a lograr mayor confluencia y homogeneidad.

Consciente de la imperiosa necesidad de evitar las evidentes falencias demostradas por acciones puntuales y sin coordinación, la nueva gestión ministerial abogó por la integralidad, entendida como "un trabajo de unificación y articulación de recursos, circuitos administrativos y gestiones compartidas y consistentes por tipo de prestaciones o beneficiarios a atender" que se tradujo en la reducción y conversión "de pequeños y grandes programas en tres grandes Planes que hacen más eficiente las prestaciones y le imprimen una impronta particular en cuanto a planificación y gestión” (Jefatura de Gabinete de Ministros, 2003:138-140).

En dicho sentido, se tendió al "abandono progresivo de la lógica programática instalada en la política social (existían 56 programas sociales con su propia administración) y la con- 
siguiente integración de los mismos en distintas líneas de acción que, de acuerdo con la especificidad propia de cada uno, conforman un conjunto de herramientas, mecanismos y prestaciones dirigidos a mejorar la calidad de vida de la familia" (Jefatura de Gabinete de Ministros, 2004:127).Así nacieron los tres grandes planes que estructuran el funcionamiento del mencionado Ministerio iniciados en 2003: el Plan Nacional de Seguridad Alimentaria "El Hambre más Urgente", el Plan Nacional de Desarrollo Local y Economía Social "Manos a la Obra” y el Plan "Familias Argentinas". El paso de los años han generado modificaciones en los mismos. En términos de lo aquí descripto es importante resaltar que, por ejemplo, el Programa "Manos a la obra” (Desarrollo Local y La Economía Social) ${ }^{20}$ busca "Implementar un sistema único y masivo de apoyo a iniciativas de economía social" (Jefatura de Gabinete de Ministros, 2003:142).

Englobar la mencionada cantidad de programas en tres grandes planes no sólo permite más eficacia en el accionar de la organización, permitiendo la planificación y articulación, sino que en términos de modelo de gestión es una iniciativa de diseño institucional que desanda el camino del trabajo por programas, promovido por la Nueva Gestión Pública. ${ }^{21}$ De todas formas, no puede afirmarse que la lógica de programas haya sido desterrada por completo; dicha lógica sigue vigente, aunque tal vez no al extremo de ampliar su número sin ton ni son.

La lógica de la integralidad se buscó alcanzar en el trabajo del mencionado ministerio pero también entre políticas sociales de diversas carteras ministeriales. En tal sentido se incluyen iniciativas como el Plan de Inclusión Previsional -PIP- que implicó la universalización de las jubilaciones y los derechos asociados a ellas -trascendiendo la prestación monetaria, para abarcar otras prestaciones como la salud-incluyendo a todos los adultos mayores que habían perdido la protección junto con su trabajo. ${ }^{22}$ Al PIP se suma una serie de planes y programas que hemos mencionado más arriba.

Para el tema educativo, destaca el Plan Progresar -dirigido a los jóvenes entre los 18 y 24 años que no trabajan, trabajan informalmente o tienen un salario menor al mínimo- que contempla un estipendio que les permita finalizar sus estudios en cualquier nivel educativo.

De igual importancia es el programa Conectar-Igualdad, que consiste en el otorgamiento de una computadora portátil a los alumnos y docentes de escuelas secundarias públicas, escuelas de educación especial e institutos superiores de formación docente. Su objetivo es poner al alcance de todos los estudiantes secundarios una herramienta indispensable para disminuir la brecha digital, considerando que las habilidades en el manejo de las TICs son parte de la alfabetización contemporánea.

Así vistas, políticas públicas de carácter universal confluyen en la integralidad, conformando un nuevo modo de regulación, donde los programas no son iniciativas aisladas, 
sino que hace parte de una iniciativa de universalización de derechos. La sinergia tiene un doble sentido: las políticas sociales entre sí, y de éstas con el régimen de acumulación basado en el mercado interno y el pleno empleo.

Es especialmente notorio el círculo virtuoso entre la AUH y la reconstrucción de la educación y salud públicas, en las que se inscriben programas como Plan Nacional de Educación Inicial, el ya mencionado Conectar-Igualdad y el Calendario Nacional de Vacunación.

Por ello es que además del papel de la AUH en la reducción del indicador Gini (que mide los coeficientes de desigualdad), ${ }^{23}$ debe valorarse su interacción positiva con las condicionalidades: se incrementó el número de chicos vacunados, los controles médicos maternales y la tasa de escolaridad aumentó en un año completo entre las familias que reciben la asignación (Klisberg, 2014).

Se pueden sumar muchos otros datos que muestran el papel inclusivo de la AUH y su fortalecimiento de la escolaridad primaria y media - p.e.la Encuesta Permanente de Hogares muestra cómo desciende el número de jóvenes en edad escolar buscando trabajo desde su implementación- pero lo que nos interesa destacar es tanto su articulación con otros programas como su lugar paradigmático en la concepción de lo social en las nuevas formas de estatalidad.

\section{Procesos de territorialización}

Para ser tales, las ya descriptas universalidad e integralidad tienen en la llegada a los confines del país uno de sus desafios más importantes. Por ello no es de extrañar la ampliación de la cobertura territorial de varios de los organismos que poseen tareas sociales. Ello también va de la mano con un fenómeno relevado por varios autores: el territorio se ha convertido en un lugar privilegiado de la expresión política.

Ciertamente estas características de las identidades populares se potencian cuando los guarismos de desocupación trepan a niveles insospechados previamente, como por ejemplo en la década de 1990 en Argentina, pero no se deben exclusivamente a éstos, sino a procesos más generales de transformación de las sociedades industriales, a los que Merklen (2005:48) denomina la "descomposición de la estructura salarial", produciendo de tal forma un "repliegue en el barrio" como respuesta de los sectores populares a tal situación.

Acorde a esta configuración de dichos sectores, la gestión de varios organismos estatales adaptó su estructura otorgándole cierta impronta territorial. Ello supuso el despliegue de agencias ministeriales (o de las agencias respectivas), que establecieran materialmente su presencia en todo el territorio nacional.

Particularmente hay cuatro desarrollos que muestran la situación a): los Centros de Referencia (CdRs) y los Centros de Integración (CICs) del Ministerio de Desarrollo Social; 
las Unidades de Atención Integral (UDAIs) de la ANSES y las Oficinas de Empleo (OE) del Ministerio de Trabajo y Seguridad Social.

Los Centros de Integración (CICs) "son espacios públicos de integración comunitaria, construidos en todo el país, para el encuentro y la participación de diferentes actores que trabajan de modo intersectorial y participativo para promover el desarrollo local en pos de la inclusión social y del mejoramiento de la calidad de vida de las comunidades" (Ministerio de Desarrollo Social). A fines 2004 se habían iniciado las obras para su localización en 280 comunidades y según datos de 2012, existen 625 CICs, de los cuales alrededor de 200 son rurales (MDS, 2010).

Los Centros de Referencia (CDRs) son espacios conformados por equipos interdisciplinarios que articulan las diversas líneas de acción del Ministerio para construir, junto con la comunidad, estrategias de desarrollo local y provincial desde un abordaje integral: participativamente y desde todas las miradas. Hacia 2006 se habían creado 23 Centros de Referencia del Ministerio, en cada una de las ciudades capitales de las provincias. En 2007 se pusieron en marcha 6 Centros de Articulación que descentralizan la gestión del Centro de Referencia de Buenos Aires, que para 2009 alcanzan los 13 Centros (MDS, 2010).

Las UDAI son oficinas para la atención del público en general, "distribuidas por todo el territorio nacional con el objetivo de estar cerca de cada ciudadano. En ellas se pueden realizar todos los trámites relacionados con la Seguridad Social y recibir asesoramiento adecuado sobre las prestaciones y servicios que brinda la ANSES" (http://www.anses.gob.ar/seccion/ delegaciones-de-anses-26, visualizado en mayo 2015). Hacia 2015 se contaban 350 de estas oficinas (ver en http://www.anses.gob.ar/seccion/delegaciones-de-anses-26).

Las OEs (que constituyen una Red conformada por más de 630 Oficinas de Empleo en todo el país) son oficinas en las cuales se brinda "acompañamiento en la búsqueda de empleo; orientación sobre el mercado de trabajo local; vinculación con cursos de formación profesional gratuitos (PROGRESAR), programas de empleo, talleres para la búsqueda de empleo y con puestos de trabajo. Además ofrecen asistencia técnica a microemprendedores". Asimismo, "También los empleadores que buscan personal para cubrir puestos vacantes encontrarán allí orientación sobre legislación laboral vigente, programas de promoción del empleo y de incentivos para la incorporación de personal (PROEMPLEAR), e información sobre la dinámica del empleo de su localidad y del país" (http://www.trabajo.gob.ar/empleo/mapa_oe.asp; visualizado en mayo 2015).

Por último, debemos mencionar que en 2008 se creó el Plan de Abordaje Integral ("Plan Ahí, ahí en el lugar"), a partir de una gestión interministerial, dirigido a poblaciones entre 2.000 y 10.000 habitantes en situación de alto riesgo social. Este constituye 
la jerarquización del Plan Mi Pueblo, desarrollado previamente, y concreta la idea de llegar a los territorios más desamparados con las agencias de los diferentes ministerios del área social de forma conjunta.

La concreción de estas iniciativas permiten formular la hipótesis, siguiendo nuevamente el análisis del Estado a partir de los atributos de estatidad propuestos por Ozslak (1978), particularmente el referido a la diferenciación estructural del Estado, que si bien en Argentina hace más de un siglo que se ha logrado ese atributo mediante la presencia en todo el territorio de las fuerzas armadas, de seguridad, de escuelas y hospitales, en esta última década la estatalidad social argentina se ha visto solidificada.

\section{Tendencia a fortalecer el protagonismo popular}

Un tema de fundamental importancia para un proyecto de inclusión social es la participación de los sectores populares. Desde 2003, la perspectiva sobre dicha temática ha cambiado. Si bien el tópico "participación” está presente en casi toda propuesta de políticas, sus contenidos varían profundamente según que tradición de pensamiento la fundamente.

En los últimos años, el Ministerio de Desarrollo Social mostró interés en la participación de diversos sectores del quehacer social (desde ONGs hasta sindicatos, pasando por fundaciones y credos religiosos) pero también desde el comienzo encaró propuestas en estrecha vinculación con las organizaciones populares. De tal forma, ya en la Memoria Detallada del Estado de la Nación de 2006 enunciaba como uno de sus objetivos: "Promover y fortalecer la participación y organización popular, mediante la herramienta de la capacitación, basada en la lógica democrática de la educación popular, desde la perspectiva de los derechos sociales y la construcción de ciudadanía plena”.

En línea con esta idea, una ex-funcionaria del área acuñó la noción de "protagonismo social": "los participantes son protagonistas del destino de la comunidad, de su proyecto colectivo, y por lo tanto hacen su aporte desde el mismo momento de gestación del proyecto de gobierno. Conceptos como compromiso y militancia están íntimamente vinculados a este protagonismo, ya que en estos casos el proyecto de vida personal, familiar y/o grupal, son parte del proyecto comunitario” (Bernazza, 2009:2).

Estas ideas se plasmaron en un conjunto de pequeñas iniciativas desplegadas en todo el territorio nacional. Un ejemplo pequeño pero paradigmático lo constituye el programa "Promotores Territoriales", que se inscribe en la lógica de fortalecimiento del protagonismo popular pero también en los otros ejes comentados en este apartado:

- Implica un tipo de participación social específico, puesto que a diferencia del rol que la Nueva Gerencia Pública le daba a la ciudadanía, aquí "se interpela a los participantes en el programa no como individuos sino como integrantes de cier- 
tos movimientos sociales.Y además, no de cualquier colectivo social, sino que en particular de aquellos movimientos que tuvieron activo involucramiento en la resistencia a las políticas neoliberales" (MDS, 2010:92).

- Instituye un intento de ir desarmando la fragmentación, puesto que el programa establece la conformación de "Unidades de Trabajo y Participación", las cuales son presentadas como una "unidad mínima en cada territorio, para generar mayor participación y organización comunitaria” (MDS, 210:80). Si bien esto tiende a morigerar la fragmentación social más que la del aparato administrativo del Estado, colabora indirectamente con ésta al provocar que el mismo tenga que interactuar con actores sociales con un mayor nivel de agregación más que con múltiples sujetos sociales dispersos.

- Favorece la territorialización del Ministerio, en tanto supone, tanto el relevamiento del territorio y el reconocimiento de los actores locales.

- Si bien no son empleados estatales en sentido estricto, llevan adelante prácticas que, al mismo tiempo que potencian la organización popular y suponen una entrada de la sociedad en el Estado, vehiculizan la política estatal.

Por lo demás, a lo largo de los años se han realizado infinidad de jornadas de capacitación y formación de líderes o voluntarios en las diferentes temáticas que aborda el Ministerio, que luego acompañaron los diferentes programas del mismo. Si bien son actividades menores comparadas con las acciones sociales más relevantes de la década estudiada, su simbolismo es tan fuerte que vale ponerlos como ejemplo del cambio de perspectiva.

Igual de simbólico, en el documento que presenta el Banco Popular de la Buena Fe (s/f:11), se establecen los objetivos del mismo, entre los cuales figura "Promover la organización popular: Trabajar juntos por una comunidad organizada. Una comunidad que sea artífice de su propia historia, con sus miembros participando organizada y activamente en la transformación social y política”.

\section{Conclusión}

Cuando la crisis económica y social de 2001 alcanzó al Estado neoliberal, un nuevo modo de regulación comenzó con el régimen emergente, en el que la reconstrucción de la estatalidad social jugó un papel muy importante.

El modo de regulación postneoliberal se basa en tres grandes principios:

- Relacionar políticas económicas con política social, recuperando el círculo virtuoso keynesiano, donde los programas sociales no sólo produzcan sinergia entre sí, sino que se dirijan también a generar transferencias monetarias a los bolsillos de los ciudadanos con el objetivo de fomentar el consumo y, con él, el mercado interno. Las políticas laborales, la estabilidad en el trabajo, las convenciones colectivas, la movilidad salarial, son complementarias de las de asistencia y, en 
última instancia, todas ellas se dirigen a recuperar el pleno empleo y a fortalecer la participación de la industria en el PBI.

- Recuperar el universalismo para que la inclusión con justicia social no se transforme en programas clientelares, sino que incorpore la noción de derechos sociales -o justicia social, para usar un significante clásico en la Argentina- a la de desarrollo económico.

Asimismo, si bien determinados instrumentos de política (por ejemplo, el PIP y la AUH) tienden a garantizar esos mínimos sociales inherentes al ejercicio de la ciudadanía, se trata también de recuperar la educación y salud pública para las clases medias. Este proceso, aun en ciernes, garantizaría que estos mínimos sociales fueran, además, de calidad, pues es sabido que la retirada de las clases medias de los bienes sociales -especialmente educación y salud- termina redundando en su deterioro, volviéndose servicios 'para pobres'.

Nueva institucionalidad del sector público que ponga en un pié de igualdad a las políticas sociales respecto de las económicas, en lugar de hacer depender aquellas de las decisiones del Ministerio de Economía. Esta es una recomendación que ha sido impulsada por distintas instituciones preocupadas por el desarrollo con equidad (V. gr. CEPAL) y llevada a la práctica en algunos de los gobiernos postneoliberales de América Latina como el de Ecuador con la creación de los gabinetes sociales. En el caso argentino, las políticas emanadas desde el Ministerio de Economía actúan en coordinación con las políticas de inclusión donde la Administración Nacional de la Seguridad Social -ANSES- ocupa un lugar central.

No se trata de que la Seguridad Social reemplace al Ministerio de Economía, sino de que ambas se refuercen, haciendo parte de una lógica diferente, donde lo político -en tanto espacio de realización democrática del destino colectivo de los pueblos- vuelva a ocupar un lugar central.

Por otra parte, las políticas de empleo y derechos universales -la AUH es uno de ellos- tienden a sustituir a la multiplicidad de programas que primó durante la hegemonía neoliberal.

En esta lógica, la seguridad social -y con ella ANSES, dependiente del Ministerio de Trabajo- recupera centralidad en tanto protección asociada al trabajo. Esta lógica incluye a la AUH pues, en su condición de derecho universal a la percepción de asignaciones familiares, quienes la reciben no lo hacen en el marco de programas de lucha contra la pobreza, sino en tanto trabajadores desempleados o informales, haciendo parte de una estrategia global basada en el pleno empleo.

Incluso el Ministerio de Desarrollo Social amplió sus funciones más allá de los planes sociales, señalando en su página web: 
Dos ejes guían la implementación de las políticas sociales en todo el país: la generación de trabajo digno y genuino mediante el fomento al desarrollo local sustentable en el marco de una economía solidaria, democrática y distributiva; y la protección y promoción de la familia como núcleo central para la organización y el desarrollo de la vida en comunidad.

(Ministerio de Desarrollo Social, s/f).

Aunque de hecho, este Ministerio es por excelencia el de la asistencia, su discurso ya no la pondrá en el centro de su accionar sino que, otra vez, la generación de trabajo pasa a ser el objetivo de su intervención; abandonándose el principio que guió a la Secretaría de Desarrollo Social en los años noventa, a saber, la asistencia a los desempleados estructurales, sin capacidad para integrarse en el mercado. Esto, a su turno, hizo necesarias modificaciones en su modo de intervención, con un despliegue territorial y vínculos con la sociedad diferente de la década previa.

En resumen, los gobiernos postneoliberales se propusieron reformular el sector público en clave latinoamericana y popular. De esta forma, transformaron una estructura estatal dirigida a apoyar la valorización financiera, a seducir al capital para que se instale en el país y a desplegar políticas asistencialistas para amortiguar el costo social del ajuste estructural; por otra que modificó la ecuación fiscal con las retenciones a las exportaciones, tendió a empoderar a los sectores populares y se dedicó a apoyar la producción industrial nacional y la integración sudamericana.

La reciente derrota electoral de este proceso abre, sin embargo, poderosos interrogantes sobre la sostenibilidad en el tiempo de los modos de regulación postneoliberales en Latinoamérica. ¿Son tan poderosos los mecanismos de trasnacionalización del capital que es imposible enfrentarlos a escala nacional? $\mathrm{O}$, en términos teóricos, ¿es posible construir modos de estatalidad diferentes en un contexto mundial dominado por regímenes de acumulación post-fordistas? La alternativa, desde la mitad del siglo pasado -según lo planteara la CEPAL en su etapa fundacional- es la construcción de mercados regionales. Sin embargo, la crisis simultánea de los gobiernos postneoliberales en el continente pareciera demostrar, una vez más, su dependencia de las oscilaciones mundiales de la economía.

Otro aspecto a tener en cuenta en el relativo agotamiento de la competitividad electoral de este modo de regulación, puede estar relacionado con las profundas mutaciones culturales de las sociedades post industriales, donde el sentido de solidaridad colectiva se ve jaqueado por la poderosa penetración de los medios masivos de comunicación que -junto a las TICs- ocupan cada vez más espacio en la vida cotidiana de los individuos, debilitando las mediaciones colectivas que otrora constituyeran el sujeto social: sindicatos, clubes, asociaciones intermedias de distinto tipo.

Estos son, sin embargo, temas de otra indagación. 
${ }^{1}$ Los conceptos de régimen social de acumulación y modo de regulación son caros a la escuela regulacionista. Con el primero se hace referencia a la valorización del capital, en tanto el segundo refiere primordialmente a las condiciones institucionales y estructurales de reproducción de la fuerza de trabajo y de la acumulación capitalista en general (Jessop, 1999; Boyer, 1986). En la actualidad, estos conceptos han trascendido ampliamente a los teóricos regulacionistas, para ser incorporados en marcos conceptuales más amplios ( p. e. Grassi, Hintze y Neufeld, 1994; Lo Vuolo, 1998, entre otros).

${ }^{2}$ Acá usaremos como sinónimos Estado de Bienestar y Estado social.

${ }^{3}$ No sobra recordar que antes de la reflexión de Marshall, ya Bismarck (1873-1890) había instituido en 1883 el modelo tripartito de seguridad social; en 1884 el seguro de enfermedad; en 1889 el de accidentes de trabajo, en 1891 el descanso dominical. La limitación del modelo bismarckiano es la limitación de su alcance a los trabajadores industriales y los empleados públicos, i.e. la protección se vinculaba al mundo del trabajo formal, no a la ciudadanía. Otros antecedentes importantes a mencionar sobre la previsión social es la Constitución Mexicana de 1917 -considerada pionera de constitucionalismo social- y especialmente su título "del Trabajo y la Previsión Social" y su artículo 123. En 1919 la firma del Tratado de Versalles, con cláusulas laborales que obligan a los países signatarios de dicho Tratado, y la creación de la OIT, son otros antecedentes destacables.

${ }^{4}$ Como funciones del Estado de Bienestar podemos considerar: 1) garantizar a los ciudadanos de un Estadonación- las condiciones de vida mínimas que le permitan ejercer los derechos y deberes que ésta demanda a sus integrantes; 2) construir un mínimo común de derechos y deberes, evitando que una excesiva fragmentación anule en la práctica las reglas comunes de convivencia que constituyen a la nación; 3) disolver la tensión entre igualdad jurídica y estratificación social y 4) garantizar la reproducción de la fuerza de trabajo a través de la provisión de bienes y servicios que funcionan como redistribución del ingreso y salario indirecto. (Laguado Duca, 2013).

${ }^{5}$ La expresión es de Robert Castel (1997) y refiere a aquellos ciudadanos que carecen de sistemas de protecciones, acercándose bastante al concepto de población vulnerable que usan otros autores.

${ }^{6}$ Bob Jessop llama Estado de Bienestar Keynesiano a la confluencia del régimen de acumulación fordista basado en la extensión del consumo masivo con el Estado social. Desde distintas ópticas teóricas hay acuerdo en que el fordismo sólo alcanza su madurez en tanto forma de valorización del capital a nivel mundial, con el Estado de Bienestar. Para una interesante aproximación desde la perspectiva marxista ver Howe (1981).

${ }^{7}$ La caracterización de los Estados Nacional Populares de la mitad del siglo XX como haciendo parte del mismo modo de regulación que el desarrollismo, es compartida por diferentes autores (Jessop; 1999; Andrenacci y Soldano, 2005; Cao, Rey y Laguado Duca, 2015). La desmercantilización de la política social y el fuerte papel regulador del Estado son algunas de sus características más destacadas.

${ }^{8}$ La dictadura cívico militar (1976-1983) significó una brutal ofensiva contra los trabajadores y una monumental concentración del ingreso. Pero, sea por la feudalización del poder entre las distintas armas o por falta de proyecto coherente, el proyecto económico liberal del ministro Martínez de Hoz, no fue acompañado de una reforma metódica de la 'cuestión social', ni de las políticas públicas en general. Al respecto se puede consultar Andrenacci y Soldano (2005) y Cao, Rey y Laguado Duca (2015).

${ }^{9}$ La conjunción de un nuevo régimen de trabajo caracterizado por lo que los sociólogos de la industria han llamado el toyotismo con la desregulación del mercado de trabajo y el capital, ha sido denominada por Jessop (1999) Estado de Trabajo Shumpeteriano.

${ }^{10}$ En este apartado se retoman cuestiones planteadas en Cao y Laguado (2014)

${ }^{11}$ No hay acuerdo en los investigadores sobre la caracterización de los regímenes postneoliberales. Su discusión sobrepasa ampliamente los límites de este escrito. Acá retomamos los postulados de Cao y Laguado: "La perspectiva "Nacional y Popular" está compuesta por un corpus de valores, abordajes, conocimiento, relatos no totalmente estructurados, en parte porque siempre fue un saber plebeyo, que no tuvo como una de sus prioridades alcanzar un estatus filosófico o científico reconocido por los espacios institucionales del establishment. A los fines de facilitar la comprensión del escrito, digamos que cuando hablamos de lo "Nacional y Popular" estamos pensando en los vectores emancipadores presentes en los gobiernos de Chávez en Venezuela, Morales en Bolivia, Correa en Ecuador, Kirchner-Fernández en la Argentina, entre otros” (Cao y Laguado Duca, 2014).

${ }^{12} \mathrm{La}$ discusión sobre el neodesarrollismo es amplia. Su más connotado exponente es Bresser Pereyra (2007), para una perspectiva crítica ver Laguado Duca (2013a).

${ }^{13}$ El Programa SUMAR amplía la cobertura del Plan Nacer, puesto que atiende no sólo a la población materno-infantil, sino que incorpora a los niños/as y adolescentes de 6 a 19 años y a las mujeres hasta los 64 años. En su lanzamiento se estimó que entre 2012 y 2015 brindará cobertura de salud a más de 9,5 millones de personas, incluyendo a 1,8 millones de niños y niñas de 0 a 5 años; 3,9 millones de adolescentes; 230 mil embarazadas y 3,8 millones de mujeres de 20 a 64 años que no tienen otra cobertura sanitaria que la que ofrece el sistema público de salud. En su origen, el Plan PROCEAR proyectó la entrega de 400 mil créditos hipote- 
carios para la construcción, ampliación, terminación y refacción de viviendas, como así también para adquirir aquellas que son construidas por el Programa a través de desarrollos urbanísticos. El Plan REMEDIAR es el mayor programa del mundo de provisión gratuita de medicamentos ambulatorios, garantizando el acceso de la población más vulnerable a los medicamentos esenciales. El Plan ARGENTINA TRABAJA promueve la creación de cooperativas, a través de las cuales los participantes en ellas reciben el cobro de un ingreso mensual por tareas en obras y mantenimiento del espacio público. Hacia 2011 la cantidad de incorporados llegó a casi 200 mil personas. El Plan CONCERTAR IGUALDAD entregó una netbook a cada uno de los estudiantes y docentes de las escuelas públicas secundarias, de educación especial, y de los institutos de formación docente. Hacia 2015 se habían entregado más de 4 millones de netbooks (Datos publicados en la página web de la Anses y en las propias páginas de los programas).

${ }^{14}$ En este apartado se retoman ideas de Cao, Laguado y Rey (2015)

${ }^{15}$ Esta forma de entender la estatalidad reconoce la perspectiva desarrollada por Ozslak (1978) para quien "la formación del estado nacional supone a la vez la conformación de la instancia política que articula la dominación en la sociedad, y la materialización de esa instancia en un conjunto interdependiente de instituciones que permiten su ejercicio. La existencia del estado se verificaría entonces a partir del desarrollo de un conjunto de atributos que definen la "estatidad" -la condición de "ser estado"-, es decir, el surgimiento de una instancia de organización del poder y de ejercicio de la dominación política. El estado es, de este modo, relación social y aparato institucional".

${ }^{16}$ Además de sostener que la AUH amplía el principio de solidaridad, Grassi (2012:24) considera que la AUH se mueve en el "camino de los sistema universales. Es decir, sistemas en los que el derecho a la protección y seguridad ante avatares no dependa de condiciones que escapan a la capacidad de incidencia de los sujetos, como es la dinámica del sistema de trabajo".

${ }^{17}$ Según Alonso y Di Costa (2011:114) “para el primer trimestre de 2007 el desempleo ya había caído a un 9,8\%”.

${ }^{18}$ En términos generales, una política pública universal puede definirse como una prestación uniforme y general financiada por el Estado, un beneficio que se confiere a todos los residentes de un país que pertenezcan a ciertas categorías preestablecidas, definidas por ley. Esto significa que el beneficio se otorga a partir de una regla objetiva de asignación y que esta asignación es para todos aquellos amparados en esa regla; sin intromisión de la subjetividad o la discrecionalidad de los funcionarios públicos y demás representantes del poder político (Piffano, 2009)

${ }^{19}$ En todo caso, Ocampo (2008) demuestra que en el mediano plazo resulta más económica la universalización de las políticas que la focalización.

${ }^{20}$ Este Programa instrumenta subsidios no reintegrables para adquirir insumos, y/o equipamiento para cooperativas, emprendimientos individuales y familiares, asociaciones de productores o empresas recuperadas $\mathrm{u}$ otras instancias pertenecientes a la Economía Social.

${ }^{21}$ La NGP -o el New Public Managemen en inglés- es el modelo de gestión pública que justificó el desmonte del Estado burocrático de corte weberiano que caracterizó a los regímenes de bienestar.

${ }^{22}$ La primera etapa de este plan, iniciado en 2005, incluyó a 2,6 millones de adultos mayores. Con la segunda etapa lanzada en 2014 se calcula que la tasa de cobertura había pasado desde el 64,8 a más del 90 por ciento (Anses, 2014).

${ }^{23}$ Según el Ministerio de Economía (2009) “considerando el Total de los Aglomerados Urbanos Relevados por la EPH, el Índice de Gini desciende, desde un valor de 0,428 ex ante, a un nivel de 0,406 bajo la implementación Amplia de la AUH, y de 0,41 en la modalidad Acotada, verificando un descenso del 5,14\% y del $4,21 \%$ en cada caso, registrándose en el NEA el mayor retroceso del indicador, que disminuye un 9,26\% en la modalidad Amplia y del 7,67\% en la Acotada". 


\section{Referencias}

Andrenacci, Luciano y Daniela Soldano. (2005) “Aproximaciones a las teorías de política social partir del caso argentino" en Problemas de política social en la Argentina contemporánea. Buenos Aires: Prometeo, pp. 17-79.

ANSES (s/f). http://www.anses.gob.ar/prestacion/asignacion-universal-por-hijo-92 Fecha de acceso: 8 de febrero de 2015.

ANSES (2014) Presentación ante la Comisión Bicameral de Control de los Fondos de la Seguridad Social. Disponible en https://drive.google.com/file/d/0BzQUku9vPMaLUEVpLXJoVTQydTQ/ view Fecha de acceso: 8 de febrero de 2015.

Alonso, Guillermo y Valeria Di Costa (2011) "Cambios y continuidades en la política social argentina, 2003 - 2010”, en Revista Aportes No 29:113-137.

Bernazza, Claudia Patricia (2009) Participación, protagonismo y representación social. Bs.As.: Mimeo.

Boyer, Robert (1986) La théorie de la régulation. Un analyse critique. Paris: La Découverte.

Bresser Pereyra, Luiz Carlos (2007). "Estado y mercado en el nuevo desarrollismo" en Nueva Sociedad, №210: 110-125.

Cao, Horacio y Arturo Claudio Laguado Duca (2014). "La renovación en las ideas sobre el Estado y la Administración Pública en Argentina" en Reforma y Democracia Revista del CLAD, 60:131-160.

Cao, Horacio, Arturo Claudio Laguado Duca y Maximiliano Rey (2015) El Estado en cuestión. Ideas y política en la administración pública argentina (1960-2015). Buenos Aires: Prometeo Editorial.

Castel, Robert (1997 [1995]). La metamorfosis de la cuestión social. Una crónica del salariado. Buenos Aires: Paidós.

CENDA, Centro de Estudios para el Desarrollo Argentino (2010) "La macroeconomía después de la convertibilidad" en CENDA: La anatomía de un nuevo patrón de crecimiento y la encrucijada actual. La economía argentina en el periodo 2002-2010. Ediciones Cara o Ceca. Buenos Aires, Argentina, pp. 15-85. 


\section{CIFRA, Centro de Investigación y Formación de la República Argentina/} ATE (2010). La asignación universal por hijo: a un año de su implementación. Documento de trabajoN $N^{\circ}$. Disponible en http://www.centrocifra.org.ar/publicacion.php?pid=24 Fecha de acceso: 6 de febrero de 2012.

Crojethovic, María y Ana Ariovich (2008). "La autogestión Hospitalaria: descentralización en el contexto de los 90. El caso del Hospital Nacional profesor doctor Alejandro Posadas". Papeles de trabajo. Revista electrónica del Instituto de Altos Estudios Sociales de la Universidad Nacional de General San Martín. ISSN: 1851-2577. Año 2, No 4, Buenos Aires, Dossier “Transformaciones de la Argentina contemporánea”. Disponible en http:// www.google.com.ar/url?sa $=\mathrm{t} \& \mathrm{rct}=\mathrm{j} \& \mathrm{q}=\& \mathrm{esrc}=\mathrm{s} \&$ source $=$ web\& $\mathrm{cd}=10 \& \mathrm{ved}=0 \mathrm{CFwQ}$ FjAJ\&url=http $\% 3 \mathrm{~A} \% 2 \mathrm{~F} \% 2 \mathrm{Fwww}$. idaes.edu.ar\%2Fpapelesdetrabajo $\% 2 \mathrm{Fpaginas} \% 2 \mathrm{FDocu}$ mentos\%2F04_2_Crotehovic_y_Ariovich.pdf\&ei=cSqgVc25HcynwASxwIPQBQ\&usg =AFQjCNFWFkHNenbu68lyk8v2HLnYkQAmIw\&sig2=9o3KX49fxakm4eL4xfLuVA $\&$ bvm $=$ bv.97653015,d.Y2I Consultada el 6 de febrero 2012.

Donzelot, Jacques (1994 [1984]). La invención de lo social. Ensayo sobre la declinación de las pasiones políticas. Bs. As.: Nueva Visión.

Filmus, Daniel (2012). “El impacto de la asignación por hijo. Un intento de desprestigiar”, en Página 12, 28 de marzo, disponible en http://www.pagina12.com.ar/diario/ elpais/1-190566-2012-03-28.html Fecha de acceso: 21 marzo de 2012.

Grassi, Estela (2003). Políticas y problemas sociales en la sociedad neoliberal. La otra década infame. Bs. As: Espacio.

Grassi, Estela (2012) «La política social y el trabajo en la Argentina contemporánea. Entre la novedad y la tradición», en e-l@tina. Revista electrónica de estudios latinoamericanos, Vol. 10, No 39:5-33. Disponible en http://iealc.sociales.uba.ar/publicaciones/e-latina/

Howe, Gary N. (1981). "Dependency Theory, Imperialism, and the Production of Surplus Value on a World Scale" in Latin. American Perspectives Vol. 8, 3/4:82-102.

Jefatura de Gabinete de Ministros de la República Argentina (varios años). Memoria Detallada del Estado de la Nación. Años 2003, 2004, 2006, 2007.

Jessop, Bob (1999). Crisis del Estado de Bienestar. Hacia una nueva teoría del Estado y sus consecuencias sociales. Bogotá: Siglo del Hombre. 
Kliksberg, Bernardo (2014). "Según un estudio: La Asignación Universal por Hijo mejoró la escolaridad” en C24N.COM, disponible en http://c24n.com/2014/11/13/ segun-un-estudio-la-asignacion-universal-por-hijo-mejoro-la-escolaridad/ Fecha de acceso: 17 diciembre de 20155.

Laguado Duca, Arturo Claudio (2011). La construcción de la 'cuestión social' El desarrollismo post-peronista en Argentina (1958-1970). Buenos Aires: Espacio Editorial.

Laguado Duca, Arturo Claudio (2013). "Rol de la Seguridad Social en las políticas de inclusión”. Ponencia presentada en el panel: Calidad e Innovación en Políticas Sociales. Séptimo Congreso Argentino de Administración Pública. 18 al 20 de septiembre, Mendoza.

Laguado Duca, Arturo Claudio (2013a). "El retorno del desarrollismo" en Revista Más Poder Local. Asuntos Públicos, Políticas y Gobierno No 16. Madrid: Fundación Ortega Marañón. www.maspoderlocal.es pp 6-16.

Lo Vuolo, Rubén (1998). ¿Una nueva oscuridad? Estado de Bienestar, crisis de integración social y democracia. Buenos Aires, CIEEP.

Marshall, Thomas H. (1997 [1950]). Ciudadanía, clase social y status. Madrid:Alianza

Merklen, Denis (2004). "Sobre la base territorial, la movilización popular y sobre sus huellas en la acción”, en Lavoratorio (publicación electrónica), año 6, no 16, pp. 46-53. Disponible en http://www.catedras.fsoc.uba.ar/salvia/lavbo/textos/lavbo16.pdf.

Ministerio de Desarrollo Social (2013). Página web del Ministerio. http://www. desarrollosocial.gov.ar/ Fecha de acceso: 7 junio 2013.

Ministerio de Desarrollo Social (2010). Políticas Sociales del Bicentenario I. Buenos Aires: Ministerio de Desarrollo Social.

Ministerio de Economía y Finanzas (2009) "La Asignación Universal por Hijo en Argentina”, Informe Económico No 70 (Nota Técnica), Buenos Aires: MEF.

Ocampo, José Antonio (2008). Las concepciones de la política social: universalismo versus focalización. Nueva Sociedad No 215, pp 36-61.

Oszlak, Oscar (1978). Formación histórica del Estado en América Latina: elementos teórico metodológicos para su estudio, Vol.1, N 3, Bs. As.: CEDES. 5-43. 
Perczyk, Jaime (2012) “La restitución de derechos" Página 12, 28 de marzo, disponible en http://www.pagina12.com.ar/diario/elpais/1-190566-2012-03-28.html.

Piffano, Horacio (2009). El sistema previsional argentino en una perspectiva comparada. Departamento de Economía, Facultad de Ciencias Económicas de la Universidad Nacional de La Plata.

Rosanvallon, Pierre (1995). La Nueva cuestión social. Repensar el Estado Providencia. Buenos Aires: Manantial.

Thwaites Rey, Mabel (2010). "Después de la globalización neoliberal: ¿Qué Estado en América Latina?” en OSAL Año XI, No 27, pp 19-43.

Williamson, John (1990), "What the Washington Consensus Means by Policy Reform”, en J. Williamson (ed.), Latin America Adjustment: Hoe Much Has Happened? Washington DC: The Institute for International Economics.

\section{Cómo citar este artículo}

Laguado, Arturo y Rey, Maximiliano: Transformaciones de la estatalidad social en el régimen de acumulación post neoliberal. Revista Perspectivas de Políticas Públicas (2016) Vol. 6, N 11:39-64. 